\title{
Análisis de la interacción en Twitter, entre los televidentes y los noticieros matinales en la ciudad de Guayaquil (2015)
}

\section{Analysis of interaction on Twitter, between viewers and morning news in the city of Guayaquil (2015)}

Maria Elizabeth Oviedo Anchundia, MBA.

Universidad Católica de Santiago de Guayaquil, Ecuador

Paola Lourdes Ulloa. Mgs.

Escuela Superior Politécnica del Litoral, Ecuador

Autor para correspondencia: helizabet@ hotmail.com

Fecha de recepción: 26 de Agosto de 2016 - Fecha de aceptación: 26 de Septiembre de 2016

Resumen: Ante una nueva era tecnológica la forma de informar de los medios de comunicación ha cambiado, hoy en día se utilizan herramientas interactivas e inclusivas para que los televidentes se involucren de manera directa con los comunicadores. Considerando estos cambios, el presente estudio tiene como objetivo analizar las relaciones entre los contenidos producidos y publicados por los usuarios de Twitter y los noticieros televisivos matinales de Guayaquil en el primer trimestre de 2015, con lo que se alcanzaron los siguientes objetivos: descubrir cuáles son los contenidos de interés producidos y enviados por los usuarios de twitter hacia los noticieros matinales en Guayaquil; verificar si la interacción entre los noticieros matinales y el público, contribuyen a sostener o incrementar el rating y determinar si los noticieros matinales tienen una comunicación bidireccional con sus seguidores en la red social Twitter. La metodología de la investigación fue cualitativa, se usó como una de las herramientas más importante la etnografía virtual, que permitió conocer de primera mano las necesidades de los televidentes en la web. Los resultados encontrados certificaron que la audiencia actualmente prefiere mantenerse en contacto con los miembros del equipo periodístico de los noticieros matinales de Guayaquil, para sentirse involucrados en el desarrollo de la información y en algunos casos porque el público quiere atención del periodista. Así mismo se pudo verificar que no existe correlación entre las noticias que se producen en la pantalla y las que aparecen en la red social Twitter, al menos no en tiempo real.

Palabras claves: internet; twitter; noticieros; comunidad; rating; periodismo; público

Abstract: Faced with a technology age, the way to inform from the media has changed, nowadays it is used interactive and inclusive tools to engage viewers in a direct way with the communicators. Considering this changes, this study aims is to analize the relation between the produced content and published content by users of twitter and the morning tv news from guayaquil in the first trimester of 2015 , with that we achieve this goals: discover what are the contents of interest, produced and submitted by users from twitter to the morning news of Guayaquil; check if the interaction between the morning news and the public, help sustain or increase the rating and determine whether the morning newscasts have a two-way communication with his followers on the social network Twitter. The methodology of the investigation was quialitative, it was used as one of the most important tools virtual ethnography, that allowed to see by first hand the needs of 
viewers on the web. The results found certified that the audience currently prefer to stay in touch with members of the team news of the morning news of Guayaquil, to feel involved in the development of information and in some cases because the public wants journalist's attention. Also it could be verified that there is no correlation between the news that occur on the screen and those in the social network Twitter, at least not in real time.

Key words: internet; twitter; news; community; classification; journalism; public

\section{Introducción}

Con el uso del internet móvil, de celulares y computadoras, en fin, el arribo de la cultura digital a la cotidianidad, los televidentes se enfrentan a una avalanche de información que es enviada mientras están sucediendo los hechos noticiosos, es decir, en tiempo real. En la actualidad, los ciudadanos tienen la facilidad de conocer más rápido hechos noticiosos, sin tener que esperar la hora en que empieza a transmitir una emisión del telediario. Además los usuarios de redes sociales como Twitter tienen la posibilidad de generar y transmitir contenidos informativos como: fotografías, videos y comentarios sobre hechos o propuestas periodísticas. Ante ello, los noticieros tienen la posibilidad de pasar por sus canales el material que envían los tuiteros.

Es justamente ese cambio en la interacción de las personas y los medios de comunicación, las prácticas sociales tecnológicas que se detecta en la interacción, lo que ha impulsado a investigar cómo se genera esta dinámica. La forma en la que se genera una renovación de los usos sociales que vincula la web con los noticieros televisivos matinales de Guayaquil. La innovación dentro de la producción de televisión y adaptación de los noticieros con el uso de las redes sociales como herramienta periodística, ha ocasionado una vinculación y aparentemente mayor aceptación por parte del televidente al comunicador actual. El éxito de esta nueva modalidad utilizada en los informativos matinales por los principales canales del país: Ecuavisa, RTS y TC Televisión, será analizado; así como los riesgos que ocasiona obtener información no verificada e inmediata de audiencia fiel que aporta con su contenido a los periodistas.

La presente investigación teórico-metodológica se desarrolló en tres grandes ejes: Contenidos publicados en Twitter, administración de las comunidades digitales y contribución al sostenimiento y/o incremento de rating. Al tratarse de una investigación innovadora, porque se indagará sobre las dinámicas que se generan en un espacio que en Ecuador no ha sido el protagonista de debates académicos, merece que se realice una exploración sobre los usos sociales y periodísticos que se generan entre los usuarios y los noticieros matinales. Además no hay artículos científicos con información en el Ecuador que proporcione este tipo de información.

Al no existir una investigación que aporte con datos sobre las prácticas comunicativas que se realizan en Twitter entre los usuarios y las cuentas de los noticieros matinales, se genera un texto académico por demás interesante porque pone sobre el escenario del debate científico este tipo de dinámicas, queson relativamente nuevas en Ecuador. Recién desde el 2010, las redes sociales registraron un repunte en el número de seguidores, solo ese año hubo un crecimiento del 2000 por ciento, y este pico se generó a partir del 11 de septiembre del mencionado año. Esa 
fecha marcó un hito en la historia de los medios sociales en el país porque al no existir señal abierta de televisión, las personas se informaban de lo que sucedía en cada ciudad del país a través de publicaciones en las redes sociales Twitter y Facebook. Ese día Ecuador enfrentó una huelga policial y hubo varios episodios de desorden civil y además se retuvo al Presidente de Ecuador, Rafael Correa, en el hospital de la Policía en Quito; por ello, resultaba importante para los ciudadanos del país andino mantenerse informados.

Con esta investigación, el periodismo de Ecuador adquiere un documento en el que se analiza una realidad vigente, porque involucra a los medios sociales, de los cuales no existe material académico que descríbala realidad nacional de los medios frente al fenómeno de la Cultura Digital. En la indagación que se ha realizado sobre la interacción de los tuiteros y los noticieros matinales se ha determinado que no hay una fusión o una inclusión del contenido de que aportan los usuarios de las redes sociales con lo que se visualiza en los noticieros matinales. Para comprobar ello, en la investigación se ha hecho un levantamiento bibliográfico, se trabajó con técnicas como la entrevista a expertos, la etnografía virtual y el focus group.

En este estudio se plantea la importancia que hay de una integración de los comentarios o publicaciones de los tuiteros a los noticieros matinales, pero también se advierte de la importancia de comprobar la noticia de los usuarios de redes sociales antes de integrarlas en los telediarios.

Al existir un documento académico que informe y sustente su tesis sobre teorías sustantivas y generales, la indagación será pertinente en la medida en que los medios de comunicación televisivos podrán tener argumentos sobre los cuales sustentar el cambio de dinámica con sus telespectadores que además los seguirlos en televisión los siguen en Twitter. Ello les va a dar la oportunidad a los medios a no registrar un bajón en el rating y a crecer en el número de personas que ven sus noticieros porque los seguidores de las redes sociales que están posteando en Twitter van a sentirse incluidos al medio y lo van a ver como un follow más cercano porque los incluye en su dinámica comunicativa.

Cuando el experto en las relaciones entre la sociedad y la tecnología, Manuel Castells (2001) se refería a la Sociedad Red, en aquella época, en Ecuador sonaba tan lejana esa realidad de una sociedad hiperconectada. En la actualidad esa es la cotidianidad de al menos el 50 por ciento de los ecuatorianos que tienen cuentas activas en redes sociales (INEC, 2014). Esta sociedad nacional no solo creció en número de habitantes sino que creció en megas y bites que pulolan en esta red en la que también se ha involucrado la población. Pero qué significa esta Sociedad Red ahora en Ecuador. El in between time es algo que no ha muchos les suena, pero que para los periodistas online es un reto marcado desde hace unos pocos años. El periodista argentino Pablo Manccini (2011) determinó que el In Between Time es el entretiempo que tienen las personas para informarse o informar. Es decir que ese espacio de tiempo que puede ser mientras los usuarios esperan el autobús, o mientras esperan el cambio de luz de un semáforo o cualquier entretiempo que tienen libre y quelo utilizan para revisar sus redes sociales e informase de qué está sucediendo.

\section{Interacción mediática}

\section{Marco Teórico}


Interacción mediática es determinante para los medios de comunicación analógicos. Un informe del diario estadounidense The New York Times publicado este año señala que solo un tercio de los usuarios de este medio de comunicación visitan la home page o portada del web site. Indican que la mayor parte de los usuarios accede a las noticias por las redes sociales Twitter y Facebook, por ello, este medio de comunicación ha puesto especial énfasis en las plataformas móviles. The New York Times es un referente en los medios de comunicación online porque es el diario con mayor número de visitas al mes y el único medio que ha logrado superar las ganancias que tenía en el impreso con su sitio web.

En el documento citado se destaca los avances alcanzados por The New York Times y el diario británico The Guardian. En el informe se señala como los dos medios lograron asumir prácticas digitales que aseguraron el crecimiento del número de lectores en la era digital, donde a criterio de Castell (2001) internet es la sociedad misma. La interacción es la base de este documento científico y para su mayor comprensión se detalló una definición otorgada del libro "La ética de las ONGD y la lógica mercantil" de los autores Luis Nieto Pereira, Adolfo Rodríguez Gil (2002) citan al sociólogo John B. Thompson. "La interacción mediática define que los participantes ya no comparten el mismo contexto espacio-temporal, lo que limita las posibilidades de comunicación, pero persiste el carácter dialógico (2002, p. 79)." Se refiere a que los partícipes ya no comparten un mismo espacio para interactuar sino que se hace el uso de medios técnicos para transmitir una información aunque esté separada (por ejemplo una conversación vía telefónica), esto genera algunas limitaciones al momento de intercambiar información entre los participantes. Sobre esta base se determinaron tres dimensiones que son: Contenidos producidos y publicados en Twitter, si esta interacción contribuye a sostener o incrementar el rating y si existe una comunicación de doble vía entre los noticieros matinales y sus seguidores.

En el diagrama tradicional donde la comunicación era unidireccional, en donde se contemplaba un emisor, el receptor, el mensaje y el canal (Weaver, 1949) en la actualidad ya no tiene más cabida, porque lacomunicación se genera a través de roles más dinámicos y de medios distintos. El especialista en cultura digital incluye en el debate académico el rol del nativo digital (1999). Cuando Piscitelli destaca el rol de la generación de los nativos digitales logra transformar el debate porque se establece el arribo de la cultura digital y de sus integrantes, que en este caso son jóvenes que han nacido en los años posteriores a 1980 y han vivido en permanente contacto con la tecnología. Esos nativos digitales y la sociedad en la que ellos interactúan comprenden, entre otros factores, la cultura digital. En esta nueva era hay prácticas sociales distintas, los receptores ya no cumplen el rol pasivo de recibir información. En la actualidad los nativos digitales son prosumidores (Scolari, 2008), es decir, además de recibir información también la generan y la transmiten. De esta forma generan una interacción continua con la sociedad, con los medios y entre los miembros de su grupo.

Pese a que el cambio en los usuarios es evidente y el in between time es cada vez más importante para los medios, existen empresas de comunicación que no han mejorado su interacción con el público. También existen quienes sí han logrado concretar una buena relación con sus usarios y de esta manera han logrado crear esta empatía y nexo con su audiencia. Uno de ellos es el diario estadounidense The New York Times. Ello le ha permito al medio tener en sus medios digitales un buen negocio. Y es justamente de ello a lo que se refiere el profesor Ramón 
Zallo de la universidad del País Vasco. El experto específico que la cultura digital es más que una serie de usos sociales o prácticas nuevas que se generan en los nuevos medios. La cultura digital como tal no solo involucra a la sociedad como conglomerado humano, sino como ente económico, porque la economía también registra repercusiones frente a los cambios de hábitos que tienen estos nativos digitales. Es por ello, que esta investigación es importante porque los medios de comunicación en los que se transmiten los noticieros matinales podrán tener directrices claras sobre cómo enfrentar estos cambios ante los nuevos hábitus (Bordieu, 1979) y poder establecer una mejor relación con sus usuarios y mejorar así la economía de sus empresas de comunicación.

\section{Administración de comunidades digitales}

Los habitus de los nativos digitales cambiaron totalmente la concepción de comunidad. Las comunidades ahora no solo están en físico para vivir o ser parte de ellas. Los colectivos al encontrarse en Internet también han generado el surgimiento de comunidades digitales. "Por ello el concepto de comunidad virtual está íntimamente ligado a la existencia de internet, entendida no como una autopista sino como una comunidad, no como un camino que va hacia cualquier sitio, sino como un $<<$ lugar $>>$ " (Salinas, 2003). De esta manera, este descripción de comunidad virtual se puede complementar con la visión de la Sociedad Red (Castells, 2001) para comenzar a describir a los usuarios de la red como una comunidad que es parte de la cultura digital (Zallo, 2013). Un colectivo que a su vez está integrado por las redes sociales, que las personas solían tener en el mundo analógico. Esto consiste en las relaciones o prácticas comunicativas que las personas tienen con sus amigos, familiares o personas con las que tienen temas de interés en común (Ugarte, 2012).

Una comunidad virtual, en definitiva, viene a ser la experiencia de compartir con otros que no vemos un espacio de comunicación. En este sentido, internet constituye una amplísima red de ordenadores (y ahora unidades móviles) que proporciona a cada uno de los usuarios individuales una voz en igualdad, o al menos una igualdad en la oportunidad para hablar, es decir, para participar en la comunidad. La atracción por la red y por la participación en este tipo de experiencias viene dada por la habilidad de la tecnología para legitimar públicamente la propia expresión y por la libertad que proporciona en relación a las barreras tradicionales del espacio y del tiempo (Salinas, 2003).

Esta amplísima red de ordenadores es la que en la actualidad constituye la cultura digital, pero en este contexto socia-económico existen administradores, que son los encargados de crear las condiciones necesarias para que las comunidades digitales se mantengan o crezcan. Generalmente, la comunidad digital tienen un interés en común, que puede ser un tema de coyuntura o su apoyo a una marca, entre otros intereses. El administrador de los colectivos digitales es el community manager, que es necesario para cada producto y en efecto, cada estación televisiva cuenta con una persona que se dedica a administrar las cuentas en los medios sociales del canal o de cada uno de los productos dtelevisivos. Pero no todos los administradores de las comunidades se dedican solo a este trabajo, por ello, las comunidades digitales, no han tenido la capacidad de respuesta, en tiempo real, que se requiere en los productos digitales.

\section{Twitter revolucionó la televisión}


Twitter es una revolución mundial en redes sociales, desde su creación ha tenido mucha acogida por muchas personas, en especial a periodistas, o personas públicas que comparten sus opiniones en esta red para que sea vistos por todos sus seguidores, esta herramienta es móvil, cualquier dispositivo electrónico que tenga acceso a internet puede utilizar esta red social para informarse. Carballar en su libro Twitter: marketing personal y profesional menciona que "Twitter es un servicio que permite compartir pensamientos, información, enlaces, etc., con cualquier usuario web, así como comunicarse directamente, de forma privada o pública, con otros usuarios de Twitter.” (Twitter: marketing personal y profesional, 2011, p. 4)

Es una interacción virtual entre personas, actualmente es una herramienta que se ha vuelto necesaria para muchos usuarios que utilizan este servicio, son interacciones en tiempo real e inmediatas para informar cualquier anuncio ya sea para todos o privado.

Gamez describe a esta red social como:

Twitter es un medio de comunicación donde podemos encontrar la materia prima para elaborar las noticias que nutran nuestro medio de comunicación, pero no podemos perder de vista que Twitter es una red libre, anónima, donde no hace falta pasar ningún filtro para opinar y donde las falsedades pueden ser nuestro gran enemigo y el de nuestro medio. (Twitter: como 140 caracteres puede ayudar a mejorar su presencia online, 2012, p. 87)

Los medios de comunicación, utilizan con mayor frecuencia esta red, que lo usan como fuente de información ante una noticia dada, sirve también para anunciar primicias o eventos noticiosos en donde el usuario en general es sin duda el más beneficiado.

\section{Sostener o incrementar el rating}

Según el Manual de planificación de medios, cuando la audiencia se expresa en cifras relativas como un porcentaje sobre la base que se indique al efecto, se denomina rating... Rating point (punto de rating) es también una expresión frecuentemente usada. A partir de la definición de rating, podemos decir que el 1\% de la audiencia es el rating point. (González Lobo \& Carrero López ,2008). La mayoría de medios, utilizan de referencia este término para medir la aceptación de sus recursos informativos en la sociedad. Esta cifra representa un porcentaje de personas que ven un programa de televisión. El rating es un factor determinante ya que evidencia la aceptación del público, y a su vez este establece el horario de un espacio televisivo y el período de tiempo en la pantalla, también es una medida de estudio del público y comprende que es lo que ellos desean consumir.

Según el libro de Julio Estremadoyro de Lecciones de Periodismo Televisivo, comenta que:

El rating es el porcentaje que resulta de considerar el universo total de los televisores existentes en una localidad y el número de aparatos que están sintonizando un programa (por ejemplo, si hay cien televisores y 18 están sintonizando un noticiero, el rating de este será de 18 puntos). También se puede medir considerando al total de televidentes. (Lecciones de Periodismo Televisivo, 2004, p. 140) 
El rating es la aceptación de un noticiero o programa de televisión dentro de un determinado público. Esto depende mucho de los horarios de emisión de un programa y de la interacción que pueda existir dentro del mismo con el público en casa.

Según IBOPE media el rating en los noticieros matinales de televisión centrados en información de la comunidad comienza a las 6:00 con 1.2 de rating y termina a las 8:30 con 4.7 rating aproximado en Guayaquil. Los noticieros del medio día en la ciudad de Guayaquil comienzan a las 12:30 con 4.1 de rating y termina a las 14:00 con 4.8 de rating aproximado en Guayaquil. Los noticieros de la tercera emisión en la ciudad de Guayaquil comienzan a las 19:00 con 6.1 de rating y termina a las 20:45 con 7.6 de rating aproximado en Guayaquil.

El aumento del rating en los programas de televisión durante un episodio se debe a la interacción del público en Twitter, ya que cada vez hay más gente disponible a Twitear con más frecuencia. Esto puede deberse a que el contenido de los programas induce a las personas a usar la red social con más frecuencia.

Medina Director de Estrategia del área de periodismo de la BBC manifiesta que no solo es importante conocer las cifras de rating, es igual de importante averiguar la aceptación de los programas que se emiten. Saber si el público está a gusto con el producto que se le ofrece en la pantalla"De ahí que la BBC lleve a cabo investigaciones de audiencias, considere todas las observaciones del público y tenga en cuenta los datos de audiencia proporcionados por BARB". (Revista Latina de Comunicación Social, 2009, p. 15)

\section{Métodos}

Siendo el principal objetivo de la presente investigación "analizar las interacciones entre los usuarios de la red social Twitter y los noticieros matinales en la ciudad de Guayaquil durante el 2015", se establece una investigación de tipo descriptiva. Según Merino (et al, 2010) el tipo de investigación descriptiva, permite describir situaciones, costumbres, hábitos y actitudes de un grupo de personas sometidas a estudio. Es por ello que al usar este tipo de investigación se logra conocer de manera exacta los sucesos relacionados a las variables sometidas a estudio, pues a más de requerir de una recolección de datos, esta predice e identifica las relaciones que existen entre dos o más variables dentro de un tiempo determinado. Es por ello que se dividen este tipo de investigación en dos categorías: longitudinales y transversales, siendo este último en el cual se enmarca el presente estudio, se basa en una sola etapa de recolección, donde se reúne toda la información necesaria para brindar una conclusión que llevará en su ejecución a una solución (Merino et al, 2010).

Por otra parte, la metodología del trabajo investigativo se desarrollará bajo un enfoque cualitativo, el cual según los investigadores Roberto Hernández, Carlos Fernández y Pilar Baptista en su libro de Metodología de la Investigación dice que "el análisis cualitativo implica organizar los datos recogidos, transcribirlos a texto cuando resulta necesario y codificarlos" (2010, p. 406).

\section{Instrumentos de Investigación}

Según los investigadores Roberto Hernández, Carlos Fernández y Pilar Baptista en su libro de Metodología de la Investigación dice que "los principales métodos para recabar datos 
cualitativos son la observación, la entrevista, los grupos de enfoque, la recolección de documentos y materiales, y las historias de vida" (2010, p. 406). Es por ello que en este trabajo se han utilizado esta metodología de la investigación, a fin de recabar la mayor cantidad de datos fieles a la realidad de los usuarios en la red social Twitter.

\section{Mecanismos de aproximación a los sujetos de la investigación}

En un principio se pensó obtener las cifras de la cantidad de usuarios de Twitter en Guayaquil por medio de internet, las cuales reflejan que en el año 2013 en el país se sobrepasó el millón de cuentas activas, de acuerdo a datos publicados ese año (Coberturas Móviles, Espinoza, 2013, visualizado el 30 de agosto de 3015). Debido a esto se desarrolló el procedimiento del libro Metodología de la investigación de Roberto Hernández Sampieri, donde se establece que uno de los principales métodos para recabar datos cualitativos son: la entrevista y los grupos de enfoque (2010, p. 406).

Para la ejecución técnica de esta investigación se desarrolló la entrevista estructurada a los directores de la emisión de los noticieros centrados en información sobre la comunidad de Guayaquil como primera etapa. La finalidad de esta entrevista era especificar cuáles son los recursos que Twitter como nueva herramienta ofrece al periodista y a la producción de contenidos noticiosos. Se procedió a buscar contactos cercanos para conseguir la entrevista en los directores de los tres medios de comunicación (Ecuavisa, RTS y TC Televisión).

La segunda etapa de investigación se complementará con la etnografía virtual la que se acercará a los sujetos de estudio a través de una investigación en el campo de acción de las prácticas comunicativas de los usuarios de Twiter, que en este caso es la propia red social. Esta investigación en la web nos permitió ver de qué manera los usuarios y las cuentas de redes sociales y los noticieros matinales interactuaban y también se registraron sucesos en los que no hubo interacción. Después de concluir con la segunda etapa de investigación se procedió a emitir el informe con las respuestas obtenidas.

\section{Herramientas de investigación}

Según los investigadores Roberto Hernández, Carlos Fernández y Pilar Baptista en su libro de Metodología de la Investigación dice que "los principales métodos para recabar datos cualitativos son la observación, la entrevista, los grupos de enfoque, la recolección de documentos y materiales, y las historias de vida" (2010, p. 406).

\section{Entrevista a especialistas}

Hernández cita a King y Horrocks (2009) ya que asegura que "la entrevista cualitativa es más íntima, flexible y abierta” (2010, p. 418). Es un encuentro en el que se puede dialogar e intercambiar información entre el entrevistador y el entrevistado. Mediante este proceso se puede obtener aclaraciones y significados sobre el objeto de observación. Hernández indica que "Las entrevistas semiestructuradas, por su parte, se basan en una guía de asuntos o preguntas y el entrevistador tiene la libertad de introducir preguntas adicionales para precisar conceptos $\mathrm{u}$ obtener mayor información sobre los temas deseados" (2010, p. 418).

La importancia de esta herramienta hace necesaria su presencia en la investigación, ya que esta permitirá conocer de manera profunda y desde el punto de vista profesional de un 
experto, la situación actual de los noticieros matinales con mayor raiting de los canales de televisión en la ciudad de Guayaquil. La entrevista semiestructurada se realizó a los Directores de los Noticieros de Ecuavisa, RTS y TC Televisión (Anexo 1), a través de un cuestionario con 10 preguntas

Población: En base a lo antes mencionado, la población para la presente herramienta, son los canales de televisión transmitidos en la ciudad de Guayaquil, los mismos que según (Centro Internacional de Estudios Superiores de Comunicación para América Latina, 2012) abarcan 12 medios.

Se seleccionó a los tres canales con mayor rating, para saber cuáles eran los noticieros matinales de televisión centrados en información sobre la comunidad de Guayaquil que tienen mayor número de espectadores, para lo cual se contactó vía correo electrónico a la empresa de encuesta de medios IBOPE media. Esta compañía respondió enviando los informes donde se puede constatar lo detallado en la tabla $\mathrm{N}^{\circ} 1$.

Tabla $\mathbf{N}^{\circ}$ 1. Canales con mayor rating en noticieros matinales de televisión en Guayaquil

\begin{tabular}{cll}
$\begin{array}{l}\text { Canales de } \\
\text { Guayaquil }\end{array}$ & Programa & $\begin{array}{l}\text { Franja } \\
\text { Horaria }\end{array}$ \\
\hline Ecuavisa & $\begin{array}{l}\text { Contacto en la } \\
\text { Comunidad }\end{array}$ & $7: 45-8: 30$ \\
RTS & $\begin{array}{l}\text { La Noticia en la } \\
\text { Comunidad }\end{array}$ & $7: 45-9: 40$ \\
Fuente: (Instituto Brasileño de Opinión Pública y Estadística) IBOPE TIME ECUADOR \\
Elaborado por las autoras
\end{tabular}

Una vez obtenidos los canales donde se realizará el estudio, se contactó a los directores de los medios y se realizó la entrevista, la cual se adjunta en el ANEXO A.

\section{Etnografía virtual}

También se realizaron grupos de enfoque a usuarios de la red social Twitter que hayan generado contenido para los noticieros matinales sobre información de Guayaquil. Hernández define a los grupos de enfoque "entrevistas grupales, las cuales consisten en reuniones de grupos pequeños o medianos" en este encuentro los miembros opinan sobre uno o varios temas en un ambiente informal y está dirigido por un moderador. El objetivo principal es analizar las respuestas que dan como grupo sobre un determinado tema.

Hernández menciona la cantidad de entrevistados en el grupo de enfoque depende del tema: "De tres a cinco personas cuando se expresan emociones profundas o temas complejos y de seis a diez participantes si las cuestiones a tratar versan sobre asuntos más cotidianos..." (2010, p.426). Por lo cual es aconsejable que lel número de los integrantes de un grupo focal no se excedan ya que se puede perder el control sobre los individuos y lo que se desea investigar.

En este proyecto de investigación se realizaron tres grupos de enfoque. El primer grupo fue de hombres, el segundo de mujeres y el tercero fue mixto. En cada uno se reunió a seis personas.

Los perfiles que cumplieron los integrantes del grupo de enfoque son: 
- Jóvenes guayaquileños, entre 18 a 28 años que viven en el sur de Guayaquil y que hayan generado contenido para los noticieros de la comunidad - hace un año o menos - de nivel socioeconómico medio.

- Mujeres guayaquileñas, entre 18 a 25 años que viven en el norte de Guayaquil y que hayan generado contenido para los noticieros de la comunidad - hace un año o menos - de nivel socioeconómico medio.

- Hombres y mujeres guayaquileños, entre 18 a 25 años que viven en el norte de Guayaquil y que hayan generado contenido para los noticieros de la comunidad hace un año o menos - de nivel socioeconómico medio.

También se revisó las cuentas de Twitter de estos canales y se logró hacer capturas de pantalla de usuarios de la red social que participaban activamente dentro de las cuentas oficiales de los medios de comunicación.

\section{Debate}

Meritxell Roca Sales, quien es doctora en Comunicación y Humanidades y licenciada en Ciencias de la Comunicación sostiene que "los consumidores están en condiciones de decidir no solo qué consumir, sino también cuándo, dónde y cómo" (Fundación Telefónica España, 2011, p. 1). Comenta que antes los receptores no generaban contenido, solo recibían la información pero en la actualidad el usuario como tradicionalmente actuaba en redes sociales no exite más y da paso al prosumidor, que es además de concumir contenidos los produce. Esta es una figura que sobresale de la teoría de ecología de medios de comunicación de Carlos Scolari (2011), en su blog Hipermediaciones. Sumado a esta nueva figura del usuario de los nuevos medios sociales se mantiene una interacción entre este y el medio de comunicación. Sin embargo, en el caso específico de nuestro estudio, los noticieros y/o canales tienen su propia cuenta de Twitter y en esta investigación determinaremos si se cumplen los objetivos anteriormente planteados.

Miquel Rodrigo Alsina en su libro "La Construcción de la noticia" explica que

Existen dos fases del proceso de construcción de la noticia: la circulación, consumo y la producción de la noticia. Por lo que respecta a la circulación, después de dibujar el panorama del mercado de la información, nos centramos en el uso de los distintos canales de la comunicación...El consumo de la noticia permite adentrarse en el estudio diacrónico de las teorías de los efectos, destacando la imagen social que se ha tenido de los medios de comunicación... La producción de la noticia cuenta como la narración de un hecho o la reescritura de otra narración, mientras que el acontecimiento es la percepción del hecho en sí o de la noticia. (2005)

Existen procesos reales e importantes para la construcción de la noticia, se llega primero a conceptualizar el hecho, luego narrarlo insertando el hecho en una cadena informativa para ligarlo a otros acontecimientos, después interpretar el hecho para generar un verdadero interés al público objetivo. Adicional dar una valoración extra por medio del informante (uso de imágenes) a los que consumen esa noticia y más que nada ubicar la noticia en una determinada sección o programa para ser emitida.

\section{Contenidos producidos y publicados en Twitter.} Contenido emitido por Usuarios. 
En el grafico 1 se evidencia el consumo de noticias que comparten los usuarios encuestados con los informativos mencionados anteriormente. De las damas entrevistadas: 1 prefiere difundir información sobre concursos que desarrollan los noticieros matinales sobre información de Guayaquil. 3 les gusta enviar más el contenido de entretenimiento. 4 se interesan por enviar información sobre las noticias de última hora. 1 prefiere proponer temas al equipo periodístico.

Mientras que los hombres 2 prefieren enviar información de concursos que desarrollan los noticieros matinales sobre información de Guayaquil. 2 escogieron enviar denuncias al equipo periodístico. 1 prefiere enviar información sobre noticias de última hora. 4 prefieren proponer temas periodísticos para que sean desarrollados en estos informativos.

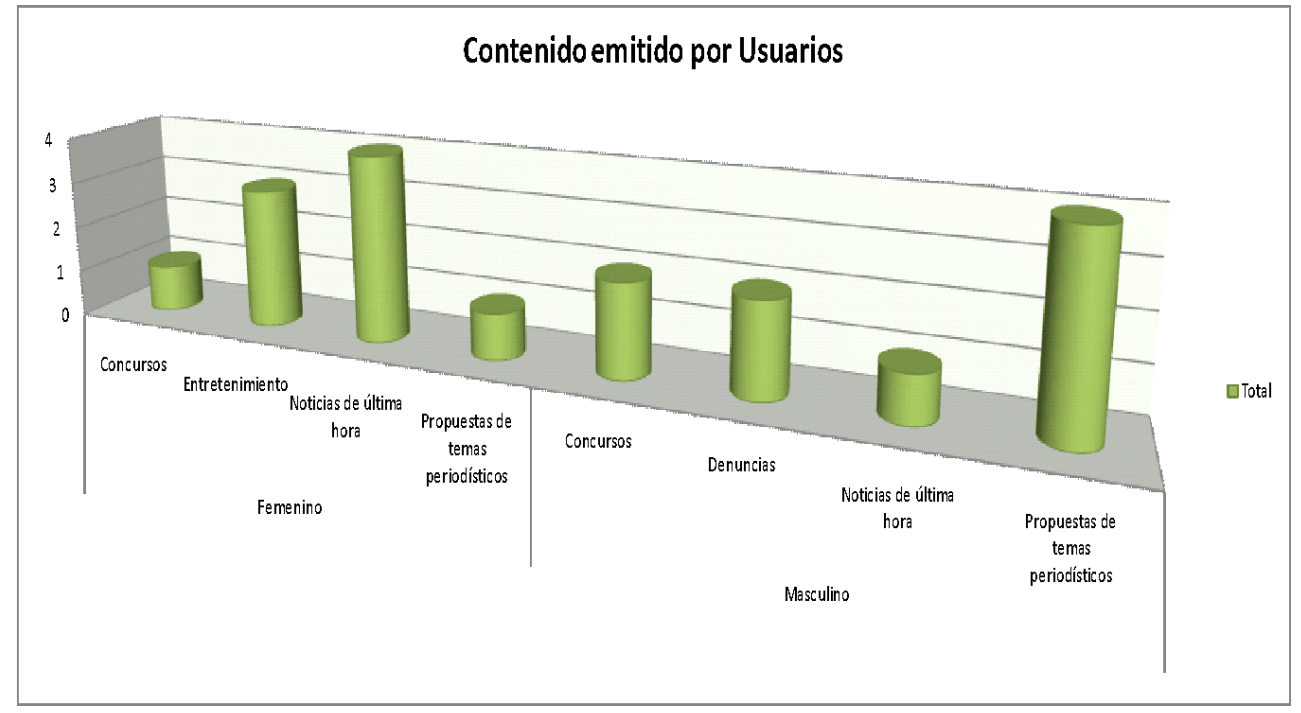

Gráfico 1. Contenido emitido por Usuarios.

Elaborado por las autoras

\section{Elaboración de la información por parte de los usuarios.}

En el grafico 2 se hace referencia al recurso que los twiteros entrevistados utilizan para la elaboración de la información y da como resultado da que 4 mujeres han usado la computadora, de esas, 2 han enviado imágenes, mientras que las otras 2 prefirieron enviar videos. En el caso de los hombres son 2 los que han utilizado la computadora para enviar comentarios.

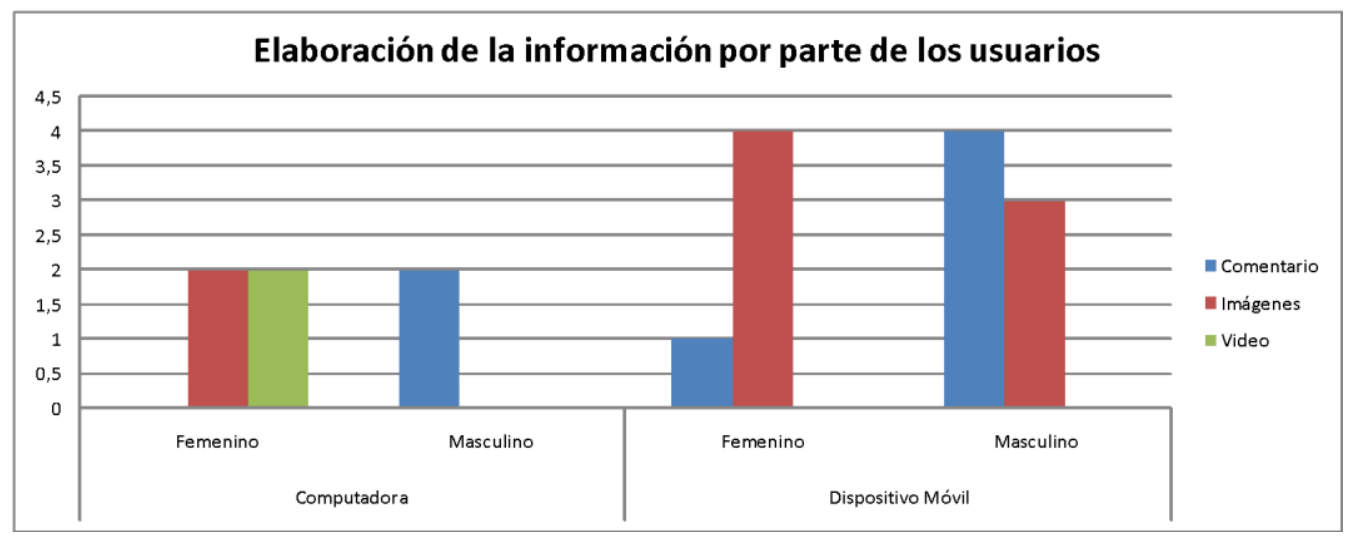




\section{Gráfico 2. Información de los usuarios}

Elaborado por las autoras

En el caso de las mujeres 5 optaron por el uso del dispositivo móvil para enviar la información elaborada por parte de los usuarios y del total 1 prefirió enviar un comentario, las otras 4 enviaron imágenes. En el caso de los hombres 7 optaron por el uso del dispositivo móvil para enviar la información elaborada por parte de los usuarios y del total 4 enviaron comentarios y 3 prefirieron enviar imágenes.

\section{Administración de las comunidades digitales}

Luego de las entrevistas con los especialistas, las estaciones televisivas no asumen a sus seguidores en Twitter como una Comunidad (Salinas, 2003) ello no permite que se establezca una empatía con las personas que los siguen en sus redes sociales. Ello permite vislumbrar que hay una deficiente administración de las comunidades digitales de los canales. Esta deficiente gestión no ha permitido poder aprovechar de mejor manera los aportes de los usuarios para que se los considere como posibles temas a tratar en los noticieros matinales. En este sentido, los nativos digitales (2009) no encuentran un nexo con los canales de televisión ni con sus noticieros matinales porque no hay una comunicación bidireccional entre los medios televisivos y sus seguidores en Twiter; por ello es cuestionable el accionar de los medios de comunicación frente a sus usuarios más jóvenes porque no hay una enculturación de la cultura digital (Zallo, 2012) de los medios analógicos. Del Fresno (2013) señala que esa actitud de los medios analógicos ha puesto en riesgo a los medios analógicos porque no han logrado percibir que el negocio cambio, ante el arribo de los nativos digitales. Ante ello, el autor señala el peligro que existe para la permanencia de las audiencias digitales como usuarios de los medios tradicionales que no han logrado cambiar ante los requerimientos de las nuevas tecnologías.

\section{Contribuye a sostener o incrementar el rating.}

Posibilidad de interactuar con los usuarios.

En Ecuavisa, Alina Manrique, ex directora encargada de Contacto en la Comunidad, indica que las denuncias no siempre se puede poner directamente al aire en tiempo real, hay que comprobar la otra parte, hay que comprobar que pasa ahí hay mucha gente que dice sin exagerar tal vez mi barrio no se puede caminar porque es demasiado inseguro, han robado 4 veces en la misma esquina, pero entonces lo que nosotros hacemos es "De esa denuncia halamos un poco el hilo y buscamos la otra parte, vamos a visitar el barrio, preguntamos a mas vecinos y moradores y preguntamos a la policía que sucede" las denuncias siempre requiere un tratamiento posterior, la información positivase publica de inmediato por ejemplo cuando el públicoenvía fotos, manda saludos, felicitaciones se menciona de inmediato en el programa; pero en cuanto a denuncias esas cosas necesitan de un tratamiento más delicado.

En RTS, Mariuxi Padilla, Directora de Noticias de LA NOTICIA EN LA COMUNIDAD, comentó que como twitter tiene solo 140 caracteres le pedimos al público que nos escriba al correo de comunidad para que nos expliquen de forma detallada su problema y luego los citamos al canal avisándoles que deben traernos toda la documentación que tengan y coordinamos como podemos ayudarlos. En TC Televisión, Carlos Armanza, Director de Noticias de Tc Televisión, señalo que se leen los mensajes, se pide una respuesta a la autoridad, y se anuncia la solución si el caso está dado por terminado adicional de que se le da seguimiento si el problema no se soluciona. 


\section{Interés del usuario.}

En el gráfico 3 muestra el interés del usuario por enviar información a los noticieros matinales centrados en información sobre la ciudad de Guayaquil. De 9 mujeres encuestadas: 6 enviaron su información con el interés de poder constatar la elaboración de un tema periodístico con su propuesta, 3 enviaron la información con el fin de merecer crédito de la información difundida por ellas.

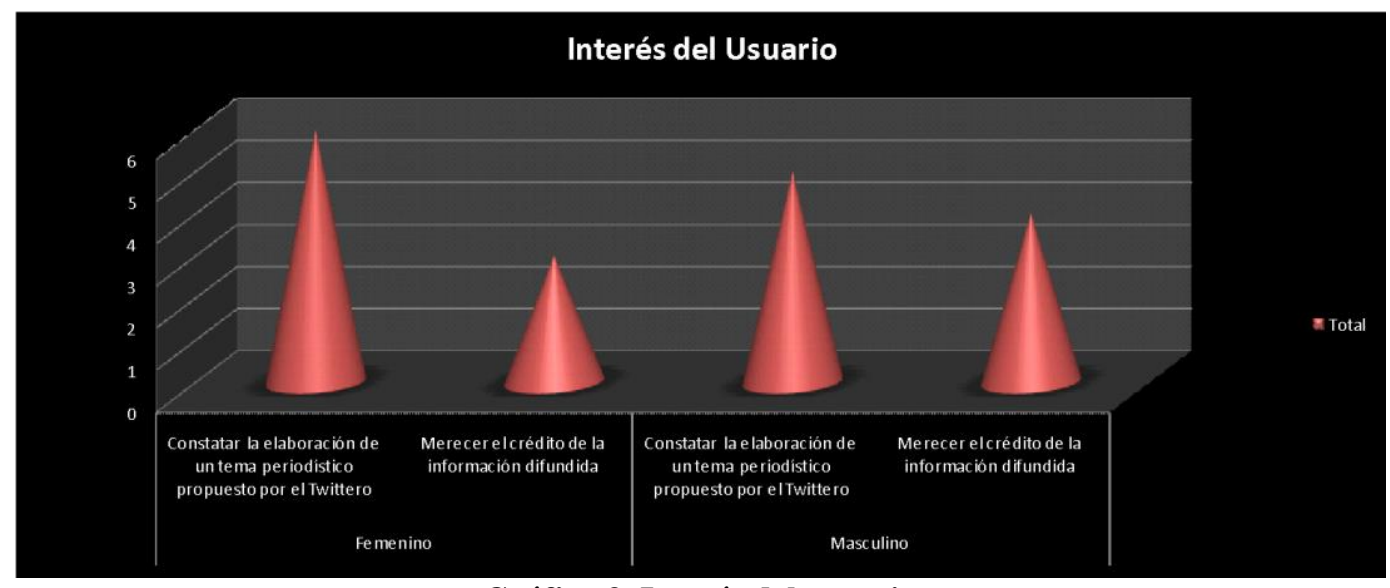

Gráfico 3. Interés del usuario

Elaborado por las autoras

De los 9 hombres encuestados: 5 prefieren enviar su información con el objetivo de constatar la elaboración de un tema periodístico en el noticiero de la comunidad, 4 enviaron la información con el interés de recibir crédito de la información proporcionada al medio de comunicación.

\section{Aumento de rating.}

En Ecuavisa, Manrique indica que aunque no todos los que los ven Contacto en la Comunidad, noticiero de Ecuavisa, tienen Twitter, si ayuda a incrementar su rating por eso es que también tratan de innovar los contenidos en esta red social, para mantenerla en el imaginario de la gente.

En RTS, Mariuxi Padilla, Directora de Noticias de LA NOTICIA EN LA COMUNIDAD, cuenta que reciben muchos tuits y que leen en vivo. Mientras que TC, Carlos Armanza, Director de Noticias de Tc Televisión, señaló que Twitter se utiliza más por ser una Red de información instantánea de interacción rápida con las autoridades. Además que actualmente la mayoría de usuarios se informan por esta vía, el uso de hashtag y herramientas de esta Red social sirven para mantener una atención considerable a los informativos en general.

Pero frente a lo expuesto por la por los directores de noticias de las tres estaciones televisivas, el colaborador de medios digitales del Centro Internacional de Estudios de Comunicación para América Latina CIESPAL, José Rivera Costales, señala que RTS es el que refleja una comunicación con menos interacción que los otros canales. "Esa cadena es la que menos utiliza redes sociales", (Rivera, 2015). El especialista señala que es común que los medios 
de comunicación de Ecuador publiquen información en sus cuentas de redes sociales sin sustento.

Además luego de hacer un monitoreo de un mes, se encontró que los noticieros matinales no tienen interacción con los usuarios y que los contenidos que se publican en las redes sociales no tienen elementos multimedios en su mayoría, es decir, no hay una producción específica para la red social Twitter. De acuerdo al monitoreo realizado en un mes se detectó, también, que los contenidos que se publican en redes sociales no son tratados en el noticiero. Un ejemplo de ello es @ comunidadgye, que durante el mes de abril y parte de mayo generó contenido en redes sociales para promocionar su concurso en el que sorteaban entradas para el concierto de Chayanne.

Mientras que en el noticiero matinal de TC Televisión no tiene una cuenta de Twitter específicamente para este programa, sino que utiliza @TCTelevisión. En esta plataforma se promocionan las telenovelas del canal, los concursos y todos los programas que se transmiten por la estación televisiva. Sumado a esta ola de promociones también se reflejan noticias que ya se han transmitido en los noticieros del canal en la noche.

En el caso de RTS, el canal de televisión ha activado la cuenta @RTS_LANOTICIA en la que solo anuncia el inicio de sus noticieros pero en la que no se hacen publicaciones con noticias nacionales o extranjeras. La investigación en la red social determinó que los canales tienen un 90 por ciento de contenidos producidos por agencias de noticias y en un 100 por ciento carecen de videos. Asimismo se determinó que una variable constante en las cuentas de Twitter de las tres estaciones televisivas es que no tienen interacción con los usuarios y que no responden las preguntas que sus usuarios realizan en línea.

Esto concuerda con lo expuesto por Rivera y el estudio que realizó sobre el uso de redes sociales en los medios de comunicación. Rivera señaló en una entrevista que se realizó para la elaboración de esta investigación que RTS tienen el manejo más deficiente de sus redes sociales. Y no destacó que TC Televisión o Ecuavisa tengan una administración aceptable de las cuentas de Twitter de los noticieros.

Estas acciones por parte de los directores de los noticieros de las estaciones televisivas de Guayaquil no contribuyen a aumentar la audiencia. Un reporte de Cobertura Móvil (2013) señala el comportamiento del consumidor nacional de contenidos en unidades móviles. Y determina que los usuarios de móviles han superado a la PC. Al menos el $26 \%$ de las cuentas de Twitter usan Web para twittear frente al de 19\% Blackberry, 10\% Android y 6\% iPhone" (es decir, 26 de escritorio frente a $35 \%$ móvil).

Estas cifras son reforzadas por el informe Immnovation, publicado este año por el diario estadounidense The New York Times, que señala que la mayor cantidad de usuarios se conecta desde plataformas móviles. En el documento se señala que este medio de comunicación alcanza los 11 millones de seguidores en Twitter, una cifra más elevada que la de sus usuarios en Facebook que suman solo 5,5 millones de usuarios. A esto se suma que lectores en web mobile suman 20 millones, mientras que lectores en el diario físico de papel apenas registra 1,25 millones de lectores. 
En Ecuavisa, la Lcda. Alina Manrique, Directora encargada de Contacto en la Comunidad, indica que twitter si los ayuda a incrementar su rating porque al público le gusta la notoriedad. En RTS la Lcda. Mariuxi Padilla, Directora del telediario Noticia en la Comunidad, que esta red social sí sirve para incrementar el rating ya que el espectador se mantiene en su sintonía esperando que su tuit sirva para generar una noticia que luego se transmita en el producto televisivo.

En Tc Televisión, el Lcdo. Carlos Armanza, Director de Nacional de Noticias, señalo que el uso de hashtag y herramientas de esta red social sirven para mantener una atención considerable a los informativos en general.

\section{Conclusiones}

Sería interesante que todos los años se realice una investigación como ésta para actualizar, contrastar y descubrir mediante el monitoreo de medios de comunicación las nuevas estrategias que utilizan los informativos de la comunidad para mantener o incrementar el rating. También se podría complementar el estudio con otras investigaciones para conocer en profundidad el tema, añadiendo factores interesantes e importantes de investigar como: Manejo de la página web de los canales de señal abierta, estrategias para incrementar el rating, etc.

Es importante que los medios de comunicación enfoquen sus estrategias de captación de usuarios a las redes sociales, especialmente Twitter, porque estudios internacionales reflejan un interesante crecimiento de seguidores de esta red social. Los Medios de Comunicación podrían establecer metas a su equipo periodístico o quienes se encargan de manejar la paginas web de sus canales para mantener un mayor tráfico en las redes sociales, incrementar la cantidad de seguidores, además de ofrecerles capacitaciones a sus trabajadores para lograr las metas propuestas y el correcto uso de redes sociales.

Es importante que los noticieros estén al tanto de que las cuentas de Twitter de su noticiero son el reflejo de su marca. Al no contestar las preguntas de usuario en la red social no se generan feedbacks y ello limita la proyección de la marca con sus seguidores o followers. De esta manera es difícil que puedan aumentar usuarios.

Las prácticas comunicativas en las redes sociales constituyen un factor determinante para los medios de comunicación si quieren que las generaciones de nativos digitales se sumen a su audiencia. Los medios deben de cambiar su estrategia on los usuarios y apropiarse de los medios sociales creando productos que involucren más a los usuarios en los productos finales que se transmiten en sus medios televisivos.

\section{Bibliografía}

Alsina, M. R. (2005). La Construcción de la noticia. Barcelona- España: Paidós Ibérica S.A.

American Psychological Association. (1972). Ethical standards of psychologists. Washington, DC: American Psychological Association. 
Anderson, C. A., Gentile, D. A., \& Buckley, K. E. (2007). Violent video game effects on children and adolescents: Theory, research and public policy. http://dx.doi.org/10.1093/acprof:oso/9780195309836.001.0001

Asne, J. H. (Mayo de 2011). ASNE. Recuperado el 2 de Julio de 2013, de http://asne.org/Files/pdf/Mejores_Practicas_SPANISH_VERSION.pdf

Beck, C. A. J., \& Sales, B. D. (2001). Family mediation: Facts, myths, and future prospects (pp. 100-102). Washington, DC: American Psychological Association. http://dx.doi.org/10.1037/10401-000

Bernstein, T. M. (1965). The careful writer: A modern guide to English usage (2nd ed.). New York, NY: Atheneum.

Bjork, R. A. (1989). Retrieval inhibition as an adaptive mechanism in human memory. In H. L. Roediger III, \& F. I. M. Craik (Eds.), Varieties of memory \& consciousness (pp. 309-330). Hillsdale, NJ: Erlbaum.

Bourdieu, P. (1979). Key Concepts. Londres y New York : Routledge.

Carlos Delgado . (2008). Versión beta: tendencias de la prensa y el periodismo del siglo XXI en Venezuela. Venezuela: Publicaciones UCAB.

Casco, M. (2010). Prácticas Comunicativas Del Ingresante. V Encuentro Nacional y II Latinoamericano La Universidad como objeto de investigación, (p. 13). Buenos Aires .

Casillas, A. M. (2003). La interacción entre las familias y la televisión. México: Editoral Aguascalientes, Ags.

Castell, M. (2001). Internet y Sociedad . La Factoría , 12.

Castells, M. (2005). La era de la información: economía, sociedad y cultura, Volume 1. Buenos Aires: Siglo Veintiuno editores .

Celaya, J. (2011). El impacto de las redes sociales y las nuevas formas de comunicación online en la estrategia empresarial. Barcelona- España: Centro Libros PAPF.

Cobo, S. (2012). Internet para periodistas, Kit de supervivencia para la era digital. Barcelona-España: UOC.

Colina, C. (2002). El lenguaje de la red: hipertexto y posmodernidad. Caracas-Venezuela: Editorial Texto. C.A.

Cress, C. M. (2009). Curricular strategies for student success and engaged learning [PowerPoint slides]. Retrieved from http://www.vtcampuscompact.org/2009/TCL_post/presenter_powerpoints/Christine\%20Cress\%2 0-\%20Curricular\%20Strategies.ppt

Digitales, Investigación Tendencias. (11 de Septiembre de 2009). Tendencias Digitales . Recuperado el 2 de Octubre de 2012, de http://www.tendenciasdigitales.com/

Driedger, S. D. (1998, April 20). After divorce. Maclean's, 111(16), 38-43. 
Espinosa, G. J. (1999). La tecnología y los sistemas de información aplicados en los negocios y la educación. San Luis Posotsí- México: Editorial Universitaria Potosina.

Estremadoyro, J. (2004). Lecciones de Periodismo Televisivo. Lima-Perú: Fondo Editorial de la Pontífica Universidad Católica del Perú.

Falcón, J. A. (2011). Twitter : marketing personal y profesional. Madrid-España: Editorial RC Libros.

Fort, D. G. (2012). Twitter: Como 140 Caracteres Puede Ayudar A Mejorar Su Presencia Online. BarceloaEspaña: Profit Editorial.

FREIRE, D. F. (2008). Las redes sociales trastocan los modelos de los medios de comunicación tradicionales. Revista Latina de Comunicación Social.

FREIRE, F. C. (2011). El nuevo escenario mediático. Zamora, España: comunicación Social S.C.

FREIRE, F. C. (2011). El nuevo escenario mediático. Zamora, España: comunicación Social S.C.

Franco, G. (2010). Cómo escribir en la web. Austin: Knight Foundation.

Fresno, M. d. (2012). El consumidor social. Reputación online y "social media". Barcelona: UOC.

Gibbs, J. T., \& Huang, L. N. (Eds.). (1991). Children of color: Psychological interventions with minority youth. San Francisco, CA: Jossey-Bass.

Gilbert, D. G., McClernon, J. F., Rabinovich, N. E., Sugai, C., Plath, L. C., Asgaard, G., ... Botros, N. (2004). Effects of quitting smoking on EEG activation and attention last for more than 31 days and are more severe with stress, dependence, DRD2 A 1 allele, and depressive traits. Nicotine and Tobacco Research, 6, 249-267. http://dx.doi.org/10.1 080/1462220041 0001676305

Goleman, D. (2009). What makes a leader? In D. Demers (Ed.), AHSC 230: Interpersonal communication and relationships (pp. 47-56). Montreal, Canada: Concordia University Bookstore. (Reprinted from Harvard Business Review, 76(6), pp. 93-102, 1998).

Guignon, C. B. (1998). Existentialism. In E. Craig (Ed.), Routledge encyclopedia of philosophy (Vol. 3, pp. 493-502). London, England: Routledge.

Gemma Gimeno, J. A. (2008). Las audiencias en la televisión y El lenguaje de las noticias de televisión. Barcelona-España: UOC.

Guembe, J. (2011). Twitter Para Dummies. Barcelona-España: Centro Libros PAPF,S.L.U.

Guembe, J. (2011). Twitter Para Dummies . Barcelona-España.

Healey, D. (2005). Attention deficit/hyperactivity disorder and creativity: An investigation into their relationship (Unpublished doctoral dissertation). University of Canterbury, Christchurch, New Zealand.

Herculano-Houzel, S., Collins, C. E., Wong, P., Kaas, J. H., \& Lent, R. (2008). The basic nonuniformity of the cerebral cortex. Proceedings of the National Academy of Sciences, 105, 12593-12598. http://dx.doi.org/10.1073/pnas.0805417105 
Hung, E. S. (2010). Tic's, comunicación y periodismo digital - Tomo I. Barranquilla: Uninorte.

Hung, E. S. (2010). Tic's comunicación y periodismo digital Tomo II, Volumen 2. Barranquilla,Colombia: Uninorte.

Instituto Nacional de Estadísticas y Censos -INEC- (2014). Ecuador en Cifras . Quito: INEC.

Jakubowicz. (2007). Revista Latina. Obtenido de http://www.revistalatinacs.org/09/art/24_823_42_ULEPICC_11/Medina_y_Ojer.html

Javier Marzal Felici, M. M. (2007). El desarrollo de la televisión digital en España. La Coruña, España: Netbiblo S.L.

Klimoski, R., \& Palmer, S. (1993). The ADA and the hiring process in organizations. Consulting Psychology Journal: Practice and Research, 45(2), 10-36. http://dx.doi.org/10.1037/10614087.45.2.10

Kubrick, S. (Director). (1980). The Shining [Motion picture]. United States: Warner Brothers.

Liu, S. (2005, May). Defending against business crises with the help of intelligent agent based early warning solutions. Paper presented at the Seventh International Conference on Enterprise Information Systems, Miami, FL. Abstract retrieved from http://www.iceis.org/iceis2005/abstracts_2005.html

Luis Nieto Pereira, Adolfo Rodríguez Gil (2002). La ética de las ONGD y la lógica mercantil. Obtenido de http://books.google.com.ec/books?id=Hfv4DQbf9mIC\&printsec=frontcover\&dq=isbn:84742661 $30 \& \mathrm{hl}=\mathrm{es} \& \mathrm{sa}=\mathrm{X} \& \mathrm{ei}=\mathrm{UKhAU} 8 \mathrm{uuE} \_$OssASfyoDACw\&ved=0CC0Q6AEwAA\#v=onepage \&q\&f $=$ false

María Ángeles González Lobo, E. C. (1997). Manual de planificación de medios. Madrid-España: Editorial Gráficas Dehon.

María Ángeles González Lobo, E. C. (2008). Manual de planificación de medios. Madrid-España: Esic Editorial.

Martini, S. (2000). Periodismo, noticia y noticiabilidad. Bogotá-Colombia: Editoral norma.

Medina, M. y. (2009). Revista Latina de Comunicación Socia. Recuperado el 3 de Octubre de 2013, de http://www.revistalatinacs.org/09/art/24_823_42_ULEPICC_11/RLCS_art823.pdf

MacIntyre, L. (Reporter). (2002, January 23). Scandal of the Century [Television series episode]. In H. Cashore (Producer), The fifth estate. Toronto, Canada: Canadian Broadcasting Corporation.

McLuhan, M. (1970a). Culture is our business. New York, NY: McGraw-Hill.

McLuhan, M. (1970b). From cliche to archetype. New York, NY: Viking Press.

Mellers, B. A. (2000). Choice and the relative pleasure of consequences. Psychological Bulletin, 126, 910924. http://dx.doi.org/10.1037/0033-2909.126.6.910

National Cable and Telecommunications Association (2010). http://www.ncta.com/Stats/BasicCableSubscribers.aspx. $\quad$ Obtenido de 
http://sociedadinformacion.fundacion.telefonica.com/DYC/TELOS/Revista/Anlisis_87TELOS_A NALISIS2/seccion=1217\&idioma=es_ES\&id=2011050916350001\&activo=7.do\#

Nielsen. (2009). Nielsen's Three Screen Report. Vol.8. Recuperado el 8 de Enero de 2013, de http://www.nielsen.com/content/dam/corporate/us/en/newswire/uploads/2010/03/3Screens_4Q09 _US_rpt.pdf

Nielsen. (20 de Marzo de 2013). New Study Confirms Correlation Between Twitter And Tv Ratings, 2013. Recuperado el 2 de Agosto de 2013, de Nielsen: http://www.nielsen.com/us/en/newswire/2013/new-study-confirms-correlation-between-twitterand-tv-ratings.html

Nielsen. (2013, March 20). The Follow-Back: Understanding The Two-Way Causal Influence Between Twitter Activity And Tv Viewership, 2013 . Retrieved August 19, 2013, from http://www.nielsen.com/us/en/newswire/2013/new-study-confirms-correlation-between-twitterand-tv-ratings.html

Noguera, J. (2012). Redes y Periodismo, Cuando las noticias se socializan. Barcelona-España: UOC.

Pérez, L. P. (2010). Periodismo zombi en la era de las audiencias participativas: La gestión participativa del público (II). Barcelona: UOC.

Postman, N. (1979). Teaching as a conserving activity. New York, NY: Delacorte Press.

Postman, N. (1985). Amusing ourselves to death: Public discourse in the age of show business. New York, NY: Viking.

Roca Sales, M. (Abril de 2011). Fundación Telefónica España. Recuperado el 2 de Julio de 2016,de https://telos.fundaciontelefonica.com/seccion=1268\&idioma=es_ES\&id=2011050916350001\&ac tivo $=6 . d o$

Sgammini, M. V. (2011). Televisión y vida cotidiana. Córdoba-Argentina: Villa María: Eduvim.

Semenak, S. (1995, December 28). Feeling right at home: Government residence eschews traditional rules. Montreal Gazette, p. A4.

Strong, E. K. Jr., \& Uhrbrock, R. S. (1923). Bibliography on job analysis. In L. Outhwaite (Series Ed.), Personnel Research Series: Vol. 1. Job analysis and the curriculum (pp. 140-146). http://dx.doi.org/10.1037/10762-000

Valcarce, D. P. (2007). De Internet 0 a Web 3.0: un reto epistemológico. Recuperado el 1 de Agosto de 2013, de http://ddd.uab.cat/pub/analisi/02112175n36p65.pdf

Ugarte, D. d. (2012). El Poder de las Redes Sociales . Madrid : El Cobre .

Zallo, R. (2011). Estructuras de la comunicación y la cultura : Políticas para la era digital. Madrid: Gedisa. 